\title{
Swertianolin ameliorates immune dysfunction in sepsis via blocking the immunosuppressive function of myeloid-derived suppressor cells
}

\author{
Zongfang Ren, $, 2,3$ Haoran Tang, ${ }^{4}$ Linjun Wan, ${ }^{3}$ Xing Liu, ${ }^{5}$ Ning Tang, ${ }^{6}$ Lingling Wang, ${ }^{2}$ Zhenhui Guo ${ }^{1,2^{*}}$ \\ ${ }^{1}$ The First School of Clinical Medicine, Southern Medical University, Guangzhou \\ ${ }^{2}$ Department of Medical Intensive Care Unit,General Hospital of Southern Theater Command of PLA,Guangzhou \\ ${ }^{3}$ Department of Critical Care Medicine, the Second Affiliated Hospital of Kunming Medical University, Kunming \\ ${ }^{4}$ Department of Gastroenterological Surgery,the Second Affiliated Hospital of Kunming Medical University, Kunming \\ ${ }^{5}$ Graduate School of Guangzhou University of Chinese Medicine, Guangzhou \\ ${ }^{6}$ Department of Neurology, The First People's Hospital of Yunnan Province, Kunming University of Science and \\ Technology, Kunming, China
}

In this study, we studied the long-term proliferation trajectory of myeloid-derived suppressor cells (MDSCs) in murine sepsis model and investigated whether swertianolin could modulate the immunosuppressive function of MDSCs. A murine sepsis model was established by cecal ligation and perforation (CLP), according to the Minimum Quality Threshold in Pre-Clinical Sepsis Studies (MQTiPSS) guidelines. The bone marrow and spleen of the mice were collected at $24 \mathrm{~h}, 72 \mathrm{~h}, 7$ and $15 \mathrm{~d}$ after sepsis induction. The proportions of monocytic-MDSCs (M-MDSCs; CD11b+LY6G-LY6Chi) and granulocytic-MDSCs (G-MDSC, CD11b ${ }^{+}$Ly6G $^{+}$Ly6C $^{\text {low }}$ ) were analyzed by flow cytometry. Then, we have investigated whether swertianolin could modulate the immunosuppressive function of MDSCs in in vitro experiments. G-MDSCs and M-MDSCs increased acutely after sepsis with high levels sustained over a long period of time. G-MDSCs were the main subtype identified in the murine model of sepsis with polymicrobial peritonitis. Furthermore, it was found that swertianolin reduced significantly interleukin-10 (IL-10), nitric oxide (NO), reactive oxygen species (ROS), and arginase production in MDSCs, while reducing MDSC proliferation and promoting MDSC differentiation into dendritic cells. Swertianolin also improved T-cell activity by blocking the immunosuppressive effect of MDSCs. Both subsets of MDSCs significantly increased in the bone marrow and spleen of the mice with sepsis, with GMDSCs being the main subtype identified. Swertianolin effectively regulated the functions of MDSCs and reduced immune suppression.

Key words: Sepsis; myeloid-derived suppressor cells (MDSCs); immunosuppression, swertianolin.

Correspondence: Zhenhui Guo, The First School of Clinical Medicine,Southern Medical University and Department of Medical Intensive Care Unit, General Hospital of Southern Theater Command of PLA, Guangzhou 510010, China. E-mail: micugzh@vip.sina.com

Contribution: ZFR, HRT, LJW, contribute equally to this study. ZHG, conceived the study and designed the experiments; ZFR, HRT, LJW, completed the experiment, analyzed the data and wrote the manuscript; XL, NT, LLW, discussed ther results and revised the manuscript. All the authors have read and approved the final version of the manuscript and agreed to be accountable for all aspects of the work.

Conflict of interest: The authors declare that they have no competing interests.

Ethical approval: Ethical approval was obtained for all experimental procedures by the Ethic Committee of General Hospital of Southern Theater Command, PLA, Guangzhou, China.

Availability of data and materials: The data and materials during the study are available from the corresponding author on reasonable request. 


\section{Introduction}

Sepsis is defined as a life-threatening organ dysfunction caused by a dysregulated immune response to infection., ${ }^{1,2}$ Approximately 27 million people worldwide develop sepsis every year, resulting in 8 million deaths. The incidence of sepsis is increasing as the population ages and the number of immunosuppressed people (cancer patients) increases. ${ }^{3-5}$ Owing to the rapid recognition and improved therapeutic and supportive care, the early survival rate of septic patients has increased, meaning that several patients survive the early stages of sepsis, normally in the first $72 \mathrm{~h}$. However, these survivors might soon show signals of immunosuppression, failure to clear the primary infection, higher susceptibility to opportunistic infections and reactivation of latent viruses.$^{6-8}$ During the development of sepsis in humans, circulating myeloid-derived suppressor cells (MDSCs) increase persistently, contributing to immunosuppression and other adverse outcomes. ${ }^{9,10}$ However, thus far, the expression trajectory of the two subtypes of MDSCs during the pathophysiological process of sepsis has not been extensively studied and the blockage of MDSCmediated immunosuppression or reducing the levels of MDSCs is expected to improve the overall outcome of sepsis.

MDSCs are a heterogenic population of immature myeloid cells consisting of myeloid progenitors and precursors of macrophages, granulocytes, and dendritic cells (DCs). ${ }^{11,12}$ Recently, two different MDSC subsets were identified in mice, according to the differences in the expression levels of LY6G and LY6C, two different epitope-specific antibodies to GR1. Granulocyte-like MDSCs (G-MDSC) have the CD11b ${ }^{+} \mathrm{LY}_{6} \mathrm{G}^{+} \mathrm{LY}^{\mathrm{l}} \mathrm{C}^{\text {low }}$ phenotype, while monocyte-like MDSCs

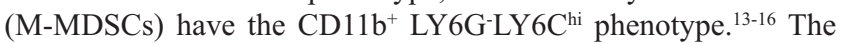
activation of these cells in a pathological context results in the production of immunosuppressive mediators, including arginase-1, inducible nitric oxide synthase (iNOS), reactive oxygen species (ROS), transforming growth factor $\beta$ (TGF- $\beta$ ), and interleukin 10 (IL-10). These mediators can inhibit T-cell proliferation and activation, and innate immune cell functions. ${ }^{17}$

Swertia species (Gentianaceae) are widely used in traditional medicine in many Asian countries such as China, India and Japan. ${ }^{18}$ Pharmacological research indicated that the plants of the Swertia genus have a wide range of biological effects, including hepatoprotective, antiinflammatory, antioxidant action, and inhibit the growth and metastasis of hepatocellular carcinoma cells. ${ }^{19,20}$ Swertianolin, a xanthone glucoside isolated from the Swertia species, is one of the representative constituents of many Swertia species, and has been reported to possess antiinflammatory, hepatoprotective, antioxidant activity while improving the immune function. ${ }^{21-23} \mathrm{CD} 34^{+} \mathrm{MDSCs}$ were firstly described in cancer patients in 1995 as the main cause of immune escape and metastasis progression. Subsequently, MDSCs have been reported in the areas of inflammation, infectious diseases, especially chronic infections, and sepsis. Since tumor immunity and sepsis immunity, particularly in the later stages of sepsis, are surprisingly similar, we speculated that swertianolin might regulate the immune response in the late stages of sepsis, by improving the functionality of MDSCs. ${ }^{12,24-27}$ In summary, our objective was to elucidate the longterm proliferation trajectory of MDSCs and explore an effective drug that can modulate the function of MDSCs.

\section{Materials and Methods}

\section{Experimental animals and late sepsis model}

Eight-week-old C57BL/6 mice (body weight ranging from 25 to $30 \mathrm{~g}$ ) were purchased from Shanghai SLAC Laboratory Animal Co. Ltd. The animals were housed in clear plastic cages with free access to water and food in an established animal house with a temperature set to $22 \pm 2{ }^{\circ} \mathrm{C}, 50 \%$ humidity atmosphere, and a 12 h light/dark cycle.

Severe sepsis group (40 mice) was induced by using a clinically relevant model of polymicrobial peritonitis caused by cecal ligation and puncture (CLP), which has been extensively used to investigate sepsis. CLP was performed as previously described. ${ }^{26,27}$ After CLP, the animals were resuscitated using an injection of prewarmed normal saline $\left(37^{\circ} \mathrm{C} ; 5 \mathrm{~mL}\right.$ per $100 \mathrm{~g}$ body weight $)$ subcutaneously. A treatment with butorphanol was used for postoperative analgesia and this was repeated every $6 \mathrm{~h}$ for at least 2 days (d). Organ/system dysfunction was assessed based on increased serum concentrations of bilirubin (liver injury marker) and creatinine (kidney function marker). Sham-operated group (20 mice) were treated identically except their cecum was neither ligated nor punctured. The experiments were performed according to the National Institutes of Health guidelines and approved by the University of Southern Medical Animal Care and Use Committee.

\section{Fluorescence-activated cell sorting staining and flow cytometry}

The bone marrow and spleen of both sepsis and sham groups were harvested after 1, 3, 7, and $15 \mathrm{~d}$. A single-cell suspension of the bone marrow or spleen was prepared by pipetting and filtering through a $70-\mu \mathrm{m}$ nylon strainer, followed by erythrocyte lysis. Then, cells were labeled with the appropriate fluorochrome-conjugated antibodies by incubation for $30 \mathrm{~min}$ at $4^{\circ} \mathrm{C}$, in staining buffer (PBS plus $2 \%$ of fetal bovine serum [FBS]). After washing, samples were analyzed using LSRII Flow Cytometer (Becton Dickinson), each sample contains $5 \times 10^{5}$ cells and data were analyzed using Flowjo software.

\section{Sepsis-surviving mice were treated with Legionella pneumophila}

$1.5 \times 10^{6} \mathrm{CFU} / \mathrm{mL}$ of Legionella pneumophila serotype 1 (CR1326) was inoculated on a BCYE plate. After $72 \mathrm{~h}$ of incubation at a constant temperature of $37^{\circ} \mathrm{C}$ with $5 \% \mathrm{CO}_{2}$ the colonies were counted. Next, the bacteria were collected and dissolved in phosphate buffer saline to a total volume of $1.25 \times 10^{9} \mathrm{CFU} / \mathrm{mL}$. On the $15^{\text {th }}$ day after CLP, sepsis-surviving mice were randomly and double-blind divided into two groups, a Legionella pneumophila treated group (LP group) and phosphate buffer saline group (PBS group). A healthy control group (HC group) was also set up in parallel. Mice in the LP group and HC group were given $40 \mu \mathrm{L}$ of suspension containing $5 \times 10^{7}$ bacterial colonies injected into the nasal cavity, respectively. In the PBS group, $40 \mu \mathrm{L}$ PBS was dropped into the nasal cavity at the same time of other two groups.

\section{Lung tissue section preparation and HE staining}

The euthanized mice in each group were fixed on the operating table, and the chest was opened to fully expose the heart and lung. The left hilum was ligated and the left lung tissue was extracted. The tissue sample was sent to the testing unit for quantitative bacterial detection within 30 min after collection. The tube was inserted into the pulmonary artery, which was then rinsed repeatedly with normal saline. After that $4 \%$ formaldehyde was injected into the artery. The right middle lobe of the lung was removed and fixed in $4 \%$ formaldehyde solution for more than $48 \mathrm{~h}$, then embedded 
in paraffin, and continuously cut into $3 \mu \mathrm{m}$ thick sections for $\mathrm{HE}$ routinary staining.

\section{Isolation and purification of MDSCs}

MDSCs were collected from the bone marrow and spleen from mice $7-10 \mathrm{~d}$ after sepsis by using the method previously described..$^{15}$ The fluorochrome-conjugated antibody FITC-GR-1 and APC-CD11b identified the MDSCs together. The LSRII Flow Cytometer (Becton Dickinson, Franklin Lakes, NJ, USA) was used to assess the purity of GR $-1{ }^{+} \mathrm{CD} 11 \mathrm{~b}^{+}$cells was $>90 \%$.

\section{ELISA}

Concentrations of IL-10, NO, ROS, and argininase in the culture supernatant were measured. ELISA kits were used in accordance to the product instructions. Each sample was tested three times. Mouse Nitric oxide (NO) ELISA Kit (WK-SU17963, Shanghai Walan Biotechnology Co., Ltd., Shanghai, China), Mouse Reactive oxygen species (ROS) ELISA Kit (WK-SU00321, Shanghai Walan Biotechnology Co., Ltd), Mouse argininase (Arg1) ELISA Kit (WK-SU22504, Shanghai Walan Biotechnology Co., Ltd.), Mouse interleukin-10(IL-10) ELISA Kit (H009-1, Nanjing Jiancheng Bioengineering Institutes, Nanjing, China) were used.

\section{MDSCs proliferation assay}

After the swertianolin treatment, MTT was used to detect the proliferation of MDSCs at 24, 48, and $72 \mathrm{~h}$ after inducing sepsis, in both the experimental and the control group.

\section{Dendritic cell (DC) flow cytometry}

The cell population characteristic phenotype of DC was CD11c. The proportion of the DC population was determined by PE-CD11C flow antibody (LSRII Flow Cytometer, Becton Dickinson)

\section{$\mathrm{CD3}^{+}$T-cell proliferation assay}

To observe the inhibitory function of MDSC cells and swertianolin on T cells, its proliferation was detected by flow cytometry (LSRII Flow Cytometer, Becton Dickinson). Briefly, after $72 \mathrm{~h}$ of culture, cells in each group were collected and PE-CD3 monoclonal antibody and CFSE were added. Then, cells were incubated in the dark at $4{ }^{\circ} \mathrm{C}$ for $30 \mathrm{~min}$, washed three times with PBS, and centrifuged at $1000 \mathrm{rpm}$ for $5 \mathrm{~min}$. The supernatant was discarded, and cells were suspendedin $200 \mu \mathrm{L}$ PBS prior to detection by flow cytometry.

\section{Statistical analysis}

The measurements were expressed as mean $\pm \mathrm{SD}, \mathrm{N} \geq 3$. And analyzed by variance analysis of factorial design data using the SPSS24 software package, with a significance level of 0.05 . Two independent sample $t$-tests were used to analyze ELISA test data and the proportion of DCs, with a significance level of 0.05 . GraphPad was used for graphic analysis.

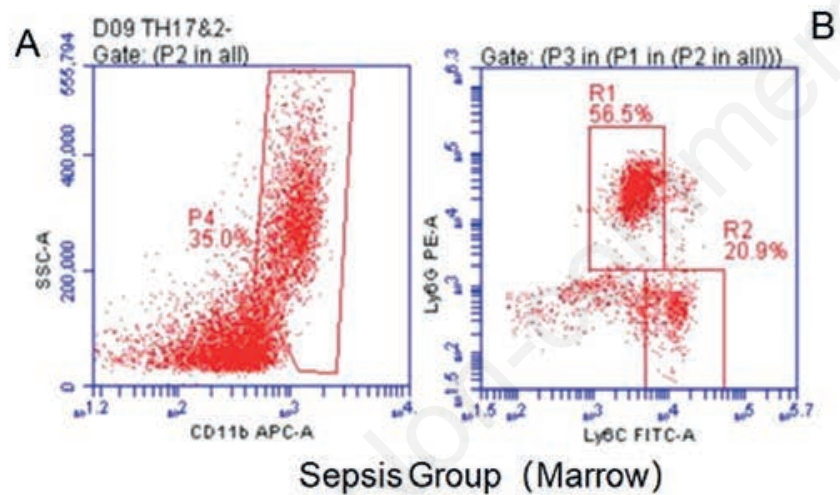

B
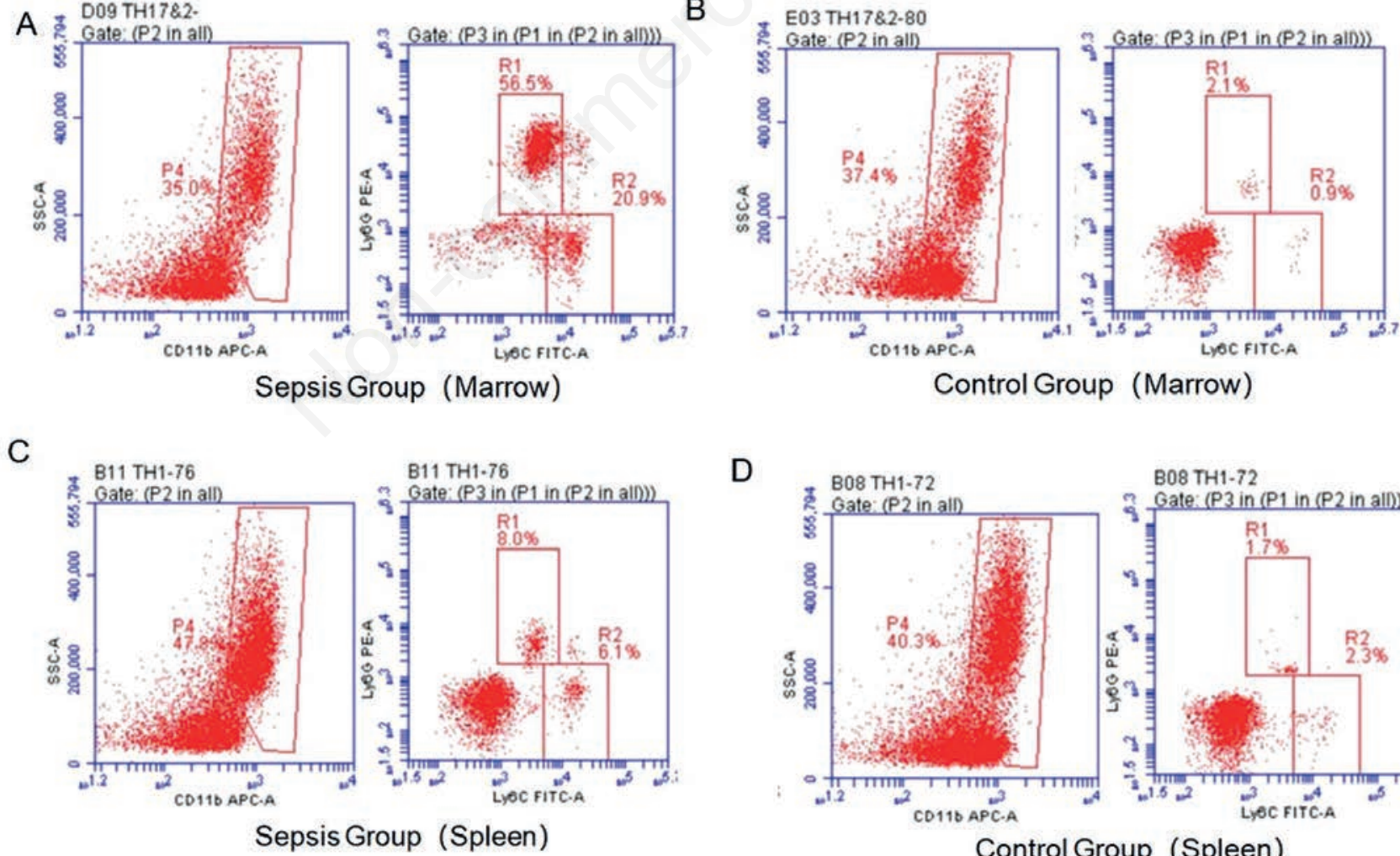

D

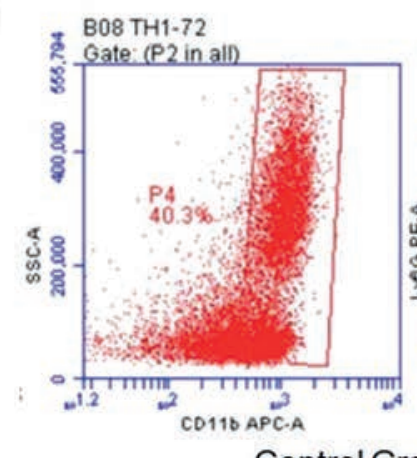

B08 TH1-72

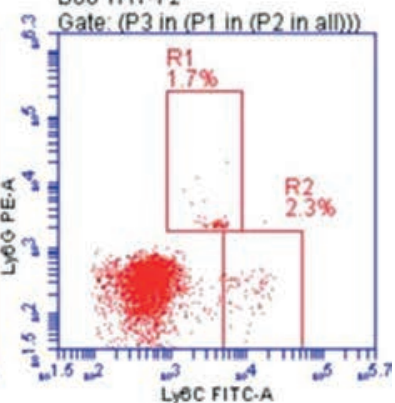

Control Group (Spleen)

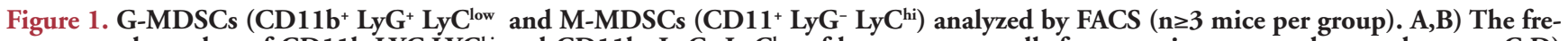
quency and number of CD11b+LYG-LYC ${ }^{\text {hi }}$ and $C D 11 b^{+} \mathrm{LyG}^{+} \mathrm{LyC}^{\text {low }}$ of bone marrow cells from sepsis group and control group. $\mathrm{C}$, D) CD11b ${ }^{+} \mathrm{LYG}^{-} \mathrm{LYC}^{\text {hi }}$ and $\mathrm{CD} 11 \mathrm{~b}^{+} \mathrm{LyG}^{+} \mathrm{LyC}^{\text {low }}$ of spleen cells from sepsis group and control group. 


\section{Results}

Acute increase and long-term maintenance of G-MDSCs and M-MDSCs after sepsis

Figure 1 shows G-MDSCs (CD11b ${ }^{+}$Ly6G ${ }^{+}$Ly6C $\left.{ }^{\text {low }}\right)$ and MMDSCs $\left(\left(\mathrm{CD} 11 \mathrm{~b}^{+} \mathrm{Ly} 6 \mathrm{G}^{-} \mathrm{Ly} 6 \mathrm{C}^{\mathrm{hi}}\right)\right.$ in marrow and spleen, analyzed by FACS ( $\mathrm{n} \geq 3$ mice per group). Both G-MDSCs and M-MDSCs increased sharply and persistently over time in the marrow and spleen in the sepsis groupm during the 15-day study period. Furthermore, their levels remained high compared to those in the control group (Figure $2 \mathrm{~A}-\mathrm{D}$ ). G-MDSCs and M-MDSCs increased rapidly in the bone marrow, on the first day after sepsis, but did not markedly increase in the spleen before the third day.

The concentration of G-MDSCs was significantly higher in the sepsis group, compared to the sham group ( $=17541.591, \mathrm{p}=0.000)$, with $28.56 \pm 0.14 \%$ and $2.54 \pm 0.14 \%$, respectively. The two groups also showed significant differences at different times $(\mathrm{F}=255.759$, $\mathrm{p}=0.000$ ). The results of multiple-comparison tests showed that the proportion of G-MDSCs was highest at $15 \mathrm{~d}$ after sepsis, with significant differences at all time points. Additionally, the concentration of G-MDSCs was significantly different in bone marrow and spleen $(\mathrm{F}=5237.541, \mathrm{p}=0.000)$. The interaction effect among all combinations was also significant $(\mathrm{p}=0.000)$, and the fitting effect of the model was very ideal. The adjustment $\mathrm{R}^{2}$ was 0.999 .

The proportion of M-MDSCs had also significantly differed between the sepsis and control groups $(\mathrm{F}=2956.242$, $\mathrm{p}=0.000)$, and the proportion of M-MDSCs within the sepsis group (8.42 \pm $0.09 \%)$ was higher than that in the control group $(1.41 \pm 0.09 \%)$. Multiple-comparison analysis showed that both groups were significantly different at different times $(\mathrm{F}=349.439, \mathrm{p}=0.000)$, although this difference was more pronounced on the seventh day after sepsis. The levels of M-MDSCs showed a significantly difference between bone marrow and spleen $(\mathrm{F}=1264.368, \mathrm{p}=0.000)$. The interaction effect among all combinations was significant $(p=0.000)$, and the fitting effect of the model was quite ideal, with an adjustment of $\mathrm{R}^{2}=0.994$.

\section{G-MDSCs are the main subtype identified in mice with polymicrobial peritonitis sepsis}

The proportions of G-MDSCs and M-MDSCs in marrow on the first day of sepsis (d1) were $41.53 \pm 0.61 \%$ and $4.40 \pm 0.65 \%$ respectively, and the proportions of G-MDSCs were about 10 times that of M-MDSCs.The G-MDSCs in the marrow of $d 3, d 7$, and $\mathrm{d} 15$ are about 3 times that of M-MDSC. At the same time, GMDSCs showed the same trend in the spleen.

G-MDSCs were the dominant cellular subtype in the bone marrow and spleen of septic mice (Figure $2 \mathrm{E}-\mathrm{F}$ ). This is consistent with previous in septic patients. ${ }^{5,28}$ The accumulation of G-MDSCs was significantly stronger than that of M-MDSCs in most animal and humans with tumors, suggesting that sepsis immunity can be similar to tumor immunity.
A

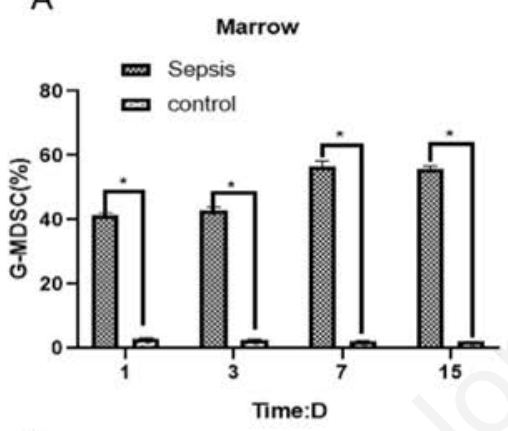

D

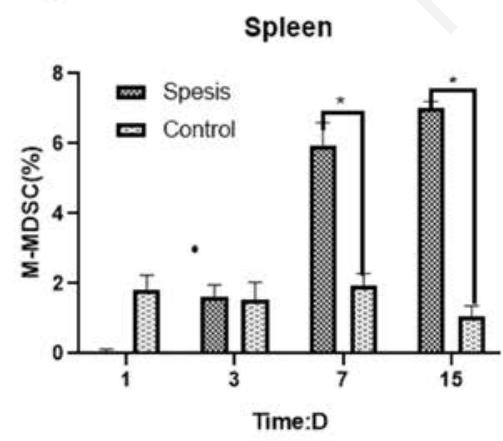

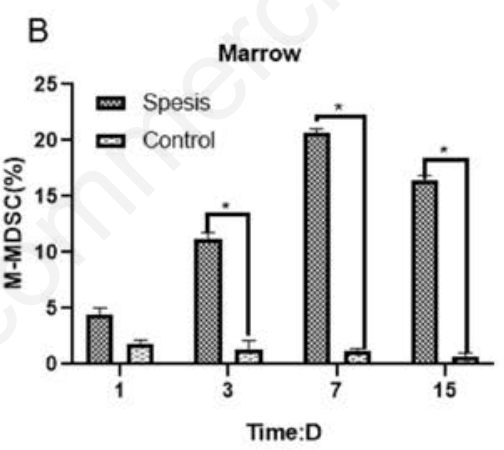

E

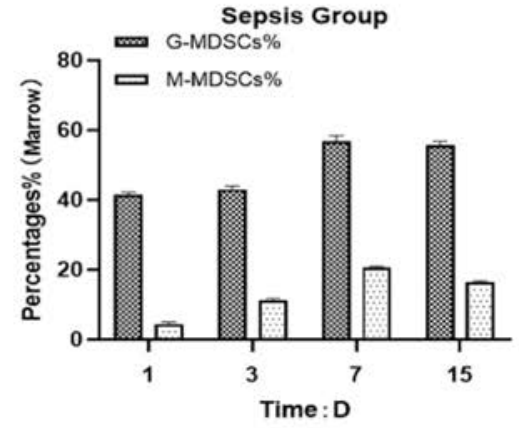

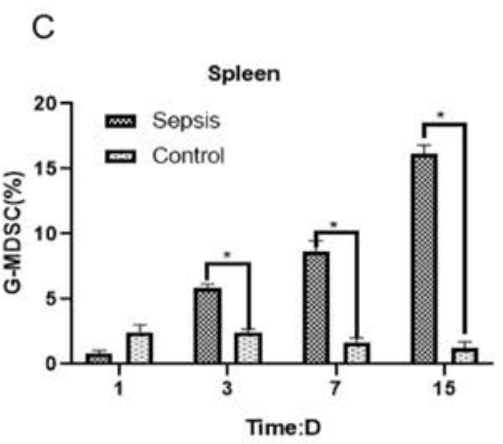

$\mathrm{F}$

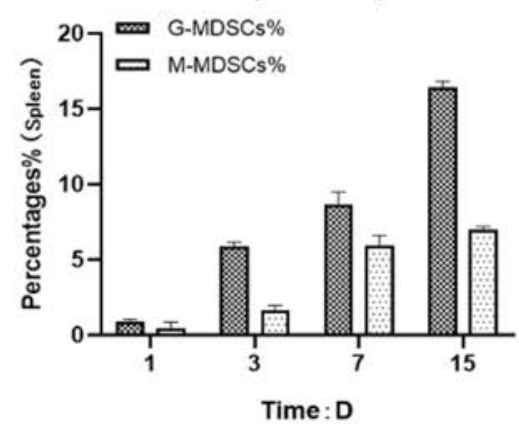

Figure 2. Percentages G-MDSCs and M-MDSCs in a murine sepsis over 15 days, ${ }^{*} \mathbf{p}<0.01$ compare to control. A) Marrow G-MDSC percentages are significantly elevated in sepsis group compared to control group at all time-points; marrow G-MDSC increased sharply on the first day after sepsis and remained at high levels. B) M-MDSC percentages in marrow are significantly elevated in sepsis group compared to control group at all time-points. C) Spleen G-MDSC percentages are dramatically increased from day 3 after CLP in sepsis group compared to control group on D3, D7, D15 with significant differences. D) Spleen M-MDSC percentages are not elevated until the third day after CLP, there was a significant difference compared to control group on D7 and D15. E,F) In sepsis group, G-MDSCs in both marrow and spleen were the dominant cellular subtype. 
Swertianolin can effectively reduce the secretion of IL10, NO, ROS, and argininase (Arg) in MDSCs

Before the formal experiment, we have first determined the most effective concentration of swertianolin for our mice model. MDSC isolated from sepsis mice was treated with different concentrations $(12.5-200 \mu \mathrm{mol} / \mathrm{L})$ of swertianolin for 24,48 , and $72 \mathrm{~h}$, before the OD 490 was detected by MTT.

Figure 3A shows that the inhibitory effect of swertianolin on MDSCs becomes more obvious with higher concentrations. However, this effect leveled off if concentrations were above 50 $\mu \mathrm{mol} / \mathrm{L}$, particularly after 48 and $72 \mathrm{~h}$, as the OD 490 did not significantly decrease with double the drug concentration. Therefore, we decided that $50 \mu \mathrm{mol} / \mathrm{L}$ was the most effective concentration for our model.

The concentration of NO in the experimental group (MDSCs + swertianolin co-culture) was $0.228 \pm 0.021 \mu \mathrm{mol} / \mathrm{L}$, while that of the control group (MDSCs culture alone) was $0.529 \pm 0.017$ $\mu \mathrm{mol} / \mathrm{L}$ (Figure 3B). Furthermore, the differences between the two groups were statistically significant $(t=-19.218, p=0.00)$. On the other hand, Figure $3 \mathrm{C}$ indicates that ROS concentration in the experimental group was of $0.193 \pm 0.01 \mu \mathrm{mol} / \mathrm{L}$ and in the control group was $0.396 \pm 0.19 \mu \mathrm{mol} / \mathrm{L}$, with a statistically significant difference between groups $(t=-15.997, p=0.00)$. Arginase was significantly depleted in the experimental group with a concentration of $0.033 \pm 0.013 \mu \mathrm{mol} / \mathrm{L}$, compared to the control group $0.444 \pm$ $0.037 \mu \mathrm{mol} / \mathrm{L}(\mathrm{t}=-17.89, \mathrm{p}=0.00)$ (Figure 3D). Finally, the concentrations of IL-10 in the experimental group and the control group were $50.28 \pm 2.38 \mathrm{pg} / \mathrm{mL}$ and $658.12 \pm 23.33 \mathrm{pg} / \mathrm{mL}$ respectively, and the difference between the two groups was statistically significant $(\mathrm{t}=-44.883, \mathrm{p}=0.00)$ (Figure 3E).

\section{Swertianolin can reduce the proliferation of MDSCs} and promote the differentiation of MDSCs into dendritic cells

The differentiation of MDSC into mature DCs was significantly higher in the experimental group than that in the control group, after treatment with swertianolin $(\mathrm{t}=36.09, \mathrm{p}=0.00)$ (Figure $3 \mathrm{~F}$ ). The mean proportion of dendritic cells was $15.04 \pm 0.39 \%$ and 3.11 $\pm 0.41 \%$ in the experiment and control groups, respectively. This means that the the proliferation of MDSCs was significantly inhibited after swertianolin treatment (Figure 3G), as the mean OD570 $(0.363 \pm 0.005)$ was significantly lower than the control $(0.626 \pm$ $0.005)$. This significance was retained across all three tested times $(\mathrm{F}=60.412, \mathrm{p}=0.000)$. The model-fitting effect was quite ideal $\left(\mathrm{R}^{2}=0.993\right)$.

MDSCs inhibited T-cell proliferation, while swertianolin improved T-cell activity by blocking the immunosuppressive effect of MDSCs

The proliferation rate of the T-cell culture alone was of $42.87 \%$ (Figure 4 A-D). The co-culture of T cells and swertianolin did not significantly promote T-cell proliferation (46.26\%), while T-cell proliferation was significantly inhibited when $\mathrm{T}$ cells were co-cul-
A
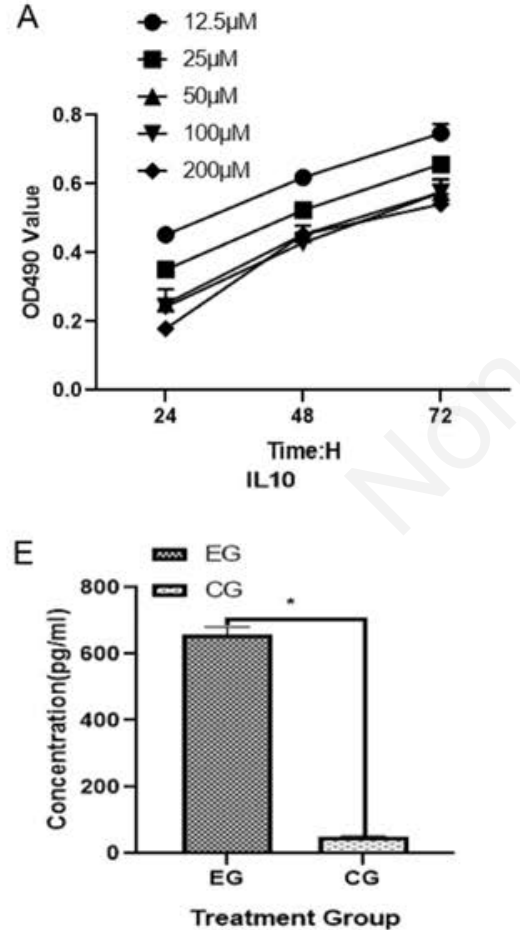

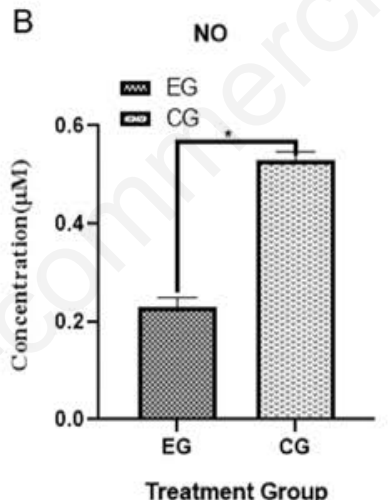

C

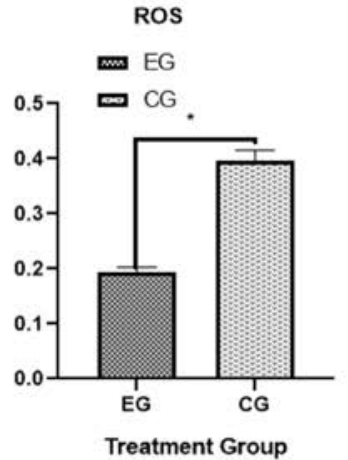

G

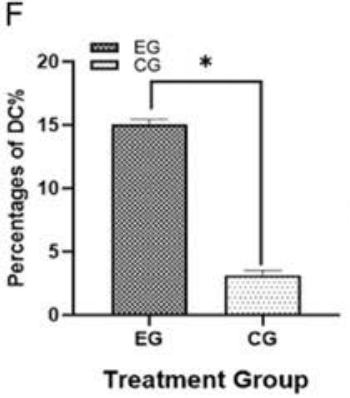

D
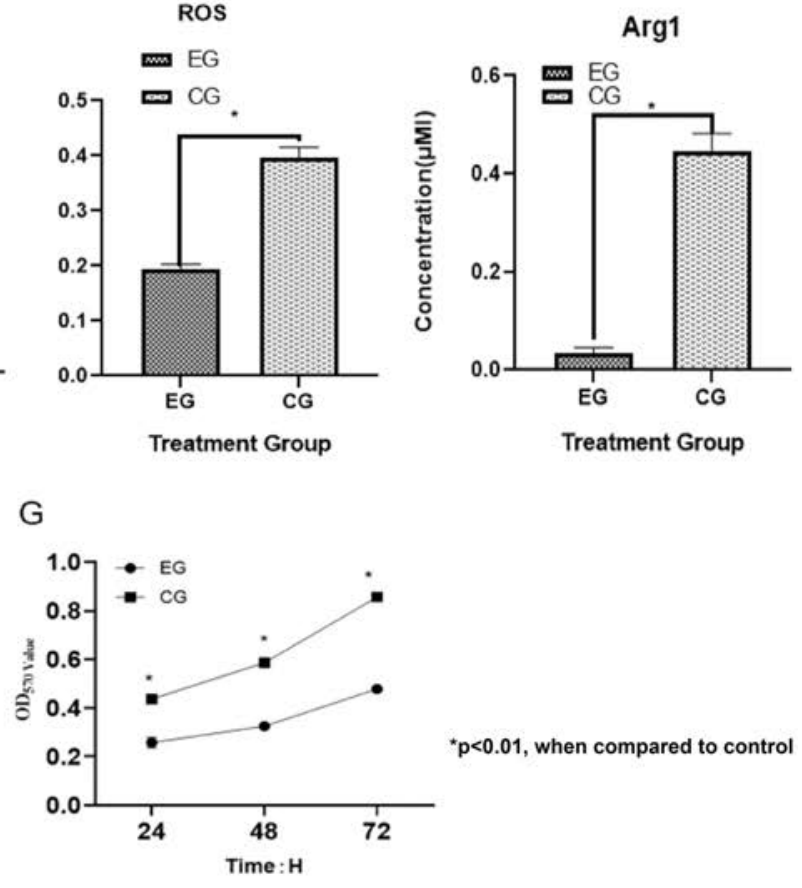

Figure 3. Effect of swertianolin on MDSC in vitro. A) MDSC were isolated from sepsis mice and treated with different concentrations $(12.5-200 \mu \mathrm{mol} / \mathrm{L})$ of swertianolin for $24,48,72 \mathrm{~h}$ then OD490 was detected by MTT. B-E) MDSCs were treated with $50 \mu \mathrm{mol} / \mathrm{L}$ swertianolin for $24 \mathrm{~h}$, concentrations of NO, ROS, Arg-1, IL-10 in the culture supernatant was tested by ELISA kit with statistically significant difference from control. F) MDSCs were treated with swertianolin $(50 \mu \mathrm{mol} / \mathrm{L})$ for $24 \mathrm{~h}$, then MDSCs were collected and the number of dendritic cells (CD11c+) were analyzed by FACS; compared with the control group, the difference was statistically significant. G) The proliferation of MDSCs was significantly inhibited after swertianolin treatment; OD 570 was significantly different between the experimental group and control groups. EG, experimental group; CG, control group. 
tured with MDSCs (21.34\%, inhibition rate of 50\%). When T cells, MDSCs, and swertianolin were co-cultured, we found that the inhibitory effect of MDSCs on T-cell proliferation was significantly decreased $(35.96 \%$, inhibitory rate of $17 \%)$. Since the stimulative effect of swertianolin on the proliferation of T cells is limited, we believe that swertianolin promoted T-cell proliferation by acting on MDSCs.

\section{Immune dysfunction in sepsis-surviving mice}

After exposure to Legionella pneumophila, HE staining of lung tissue of mice in the health control group showed a thin alveolar wall, well inflated alveolar cavity, and no exudate in the alveolar cavity (Figure 5A). In the PBS group, mice showed inflammatory exudation in alveolar cavity, and some air-filled alveoli were visible in the field of vision (Figure 5B). In contrast, in the LP group mice showed thickening of lung septum, edema of alveolar wall, large amount of inflammatory exudate in lumen, and almost no inflatable alveoli in field of vision (Figure 5C).

A

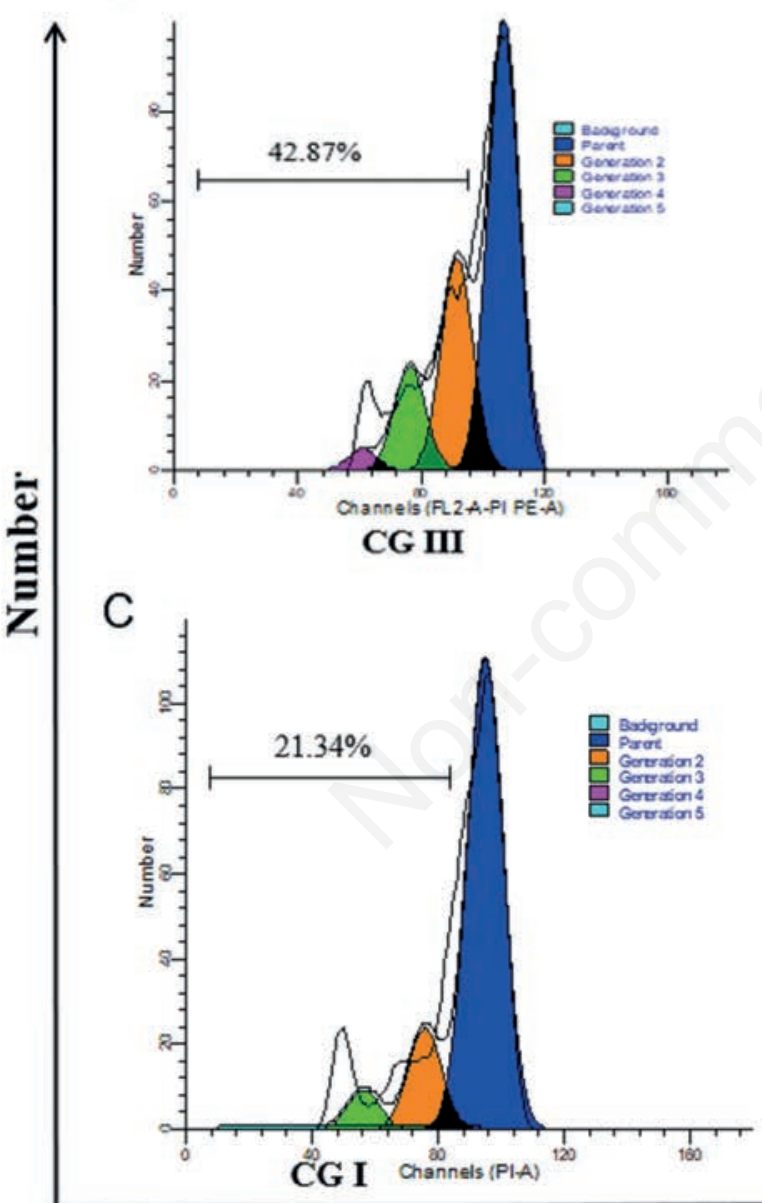

\section{Discussion}

Sepsis is not a specific disease, but a pathological syndrome involving complex immune responses with tissue and organ damage. ${ }^{29.30}$ Although infection triggers sepsis, even after the successful treatment of infection, the immune response caused by the invading pathogen often fails to return to homeostasis. ${ }^{31-33} \mathrm{To}$ enable the study of the entire pathophysiological course of sepsis and the sepsis survivors, to the mice it was administered an intraperitoneal injection of imipenem cilastatin sodium (Merck Sharp \& Dohme Corp., Kenilworth, NJ, USA; $25 \mathrm{mg} / \mathrm{kg}$ ) and fluid resuscitation $\left(0.9 \% \mathrm{NaCl}\right.$ solution $37^{\circ} \mathrm{C} ; 5 \mathrm{~mL} / 100 \mathrm{~g}$ of body weight) after the onset of sepsis. The death pattern in sepsis shows a three-way distribution. Early death is mainly due to incomplete fluid resuscitation or acute respiratory and circulatory failures. With improvements in early identification of sepsis and level of organ function support, the death rate of sepsis has decreased significantly. ${ }^{34-36}$ Currently, the peak of deaths happens mostly after
B

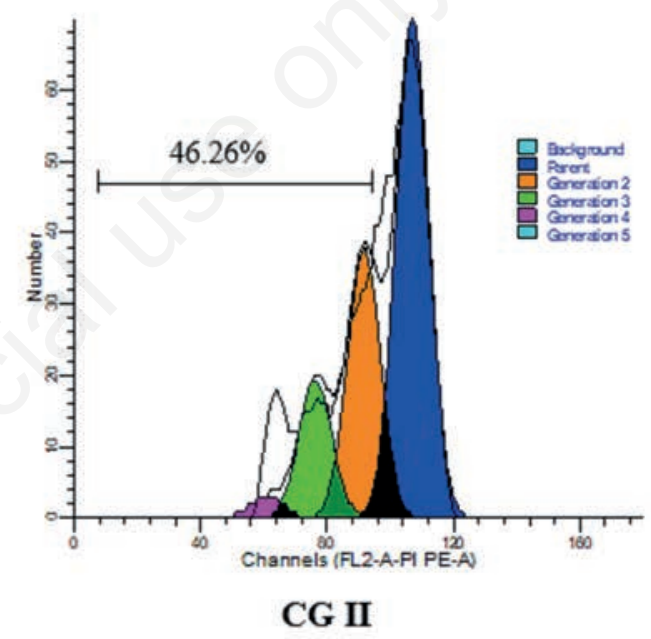

D

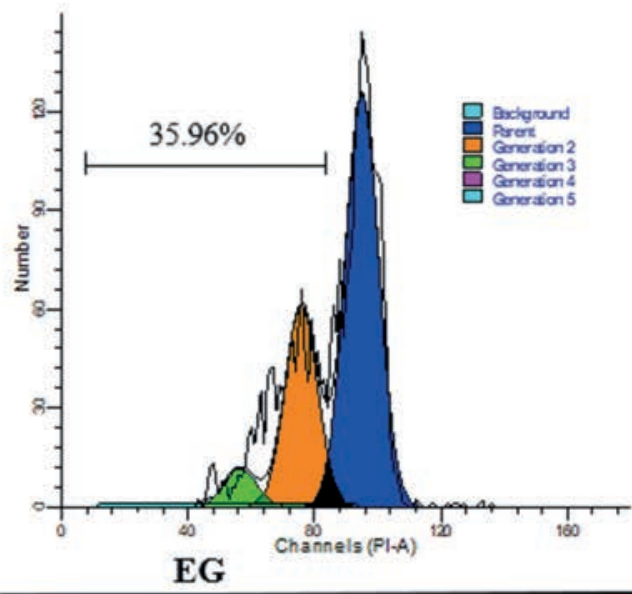

\section{CFSE}

Figure 4. Effect of swertianolin on T cells proliferation in vitro. A) CD3+Tcells were cultured alone for $72 \mathrm{~h}$, cell proliferation was determined by CFSE labeling; the proportion of $\mathrm{CD}_{3}{ }^{+} \mathrm{T}$ cell proliferation was $42.87 \%$. B) CD3+Tcells + Swertianolin (50 $\left.\mu \mathrm{mol} /\right)$ cocultured $72 \mathrm{~h}$, the proportion of proliferating $\mathrm{CD}_{3}{ }^{+} \mathrm{T}$ cells had no significant change compare to CG III (46.26\%). C) $\mathrm{MDSCs}+\mathrm{CD}_{3}{ }^{+} \mathrm{Tcells}$ cocultured $72 \mathrm{~h}$, compared with CG III and CG II, the proportion of T cell proliferation (21.34\%) was dramatically reduced. D) Swertianolin $(50 \mu \mathrm{mol} / \mathrm{L})+\mathrm{MDSCs}+\mathrm{CD}_{3}{ }^{+}$Tcells cocultured $72 \mathrm{~h}$, compared with CG I, the number of $\mathrm{T}$ cell proliferation $(35.96 \%)$ was significantly increased. 
60 to $90 \mathrm{~d}$, the main cause of late death was sepsis induced immunosuppression, and MDSCs were the main culprit of immunosuppression. ${ }^{37-39}$ Iatrogenic interferences and the inability to obtain specimens of immune organs such as bone marrow and spleen are some of the shortcomings of the clinical research that focuses on the role of MDSC in sepsis. In order to overcome this, we have established an animal model for research.

In our study, the populations of cells with strong immunosuppressive function (G-MDSCs and M-MDSCs) increased dramatically during the whole process of sepsis. To our surprise, high levels of G-MDSCs and M-MDSCs persisted in the marrow and spleen of mice that survived $15 \mathrm{~d}$ after antibiotic and fluid resuscitation treatment. The levels of G-MDSCs and M-MDSCs increased sharply in bone marrow within $24 \mathrm{~h}$ after onset of sepsis, with that of the spleed only peaking after $72 \mathrm{~h}$. This may be due to the fact that bone marrow, as a central immune organ, is quickly mobilized to generate a large number of immature immune cells after a severe infection. The late increase of G-MDSCs and MMDSCs in the spleen may indicate the presence of immunosuppression, as this occurred on a later stage of sepsis. Mice are naturally resistant to Legionella pneumophila, but using Legionella pneumophila to challenge the surviving sepsis mice again, the lungs of the mice have obvious damage, which indicates that the immune function of the surviving sepsis mice is insufficiency.

MDSCs acts as an inhibitor through a variety of mechanisms including the up-regulation of arginase, nitric oxide synthase (iNOS), and production of ROS and IL-10. ${ }^{40-42}$ Swertianolin, a natural product, showed to be a strong anticancer and antioxidant activities in both tumor and chronic infection experimental systems. ${ }^{18-23}$ Therefore, we tested the hypothesis that swertianolin may affect MDSCs. In in vitro experiments, swertianolin reduced significantly the secretion of immunosuppressor factors (IL-10, NO, ROS, argininase). Additionally, it promoted an effective differentiation of MDSCs into DCs.

\section{A}

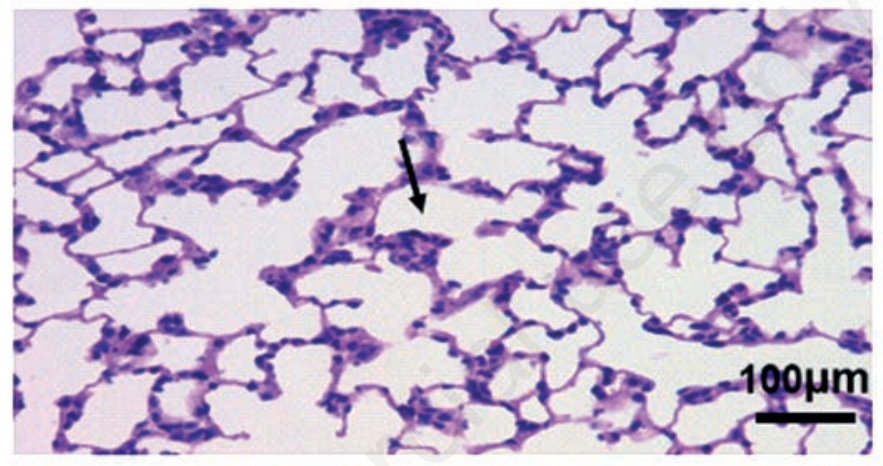

B

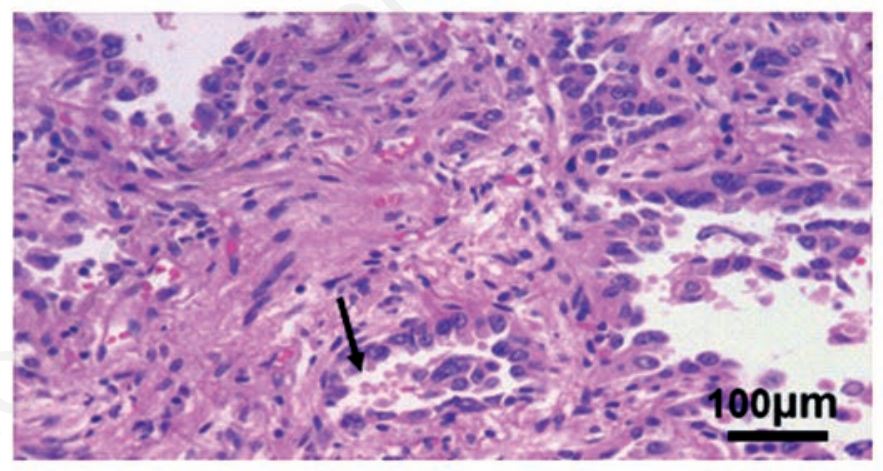

$\mathrm{C}$

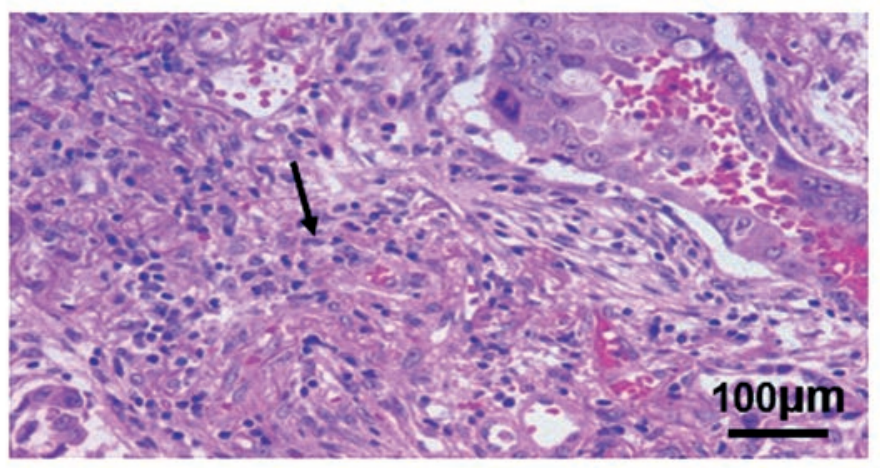

Figure 5. After a Legionella pneumophila challenge, HE staining images showing lung tissues of mice in healthy control group, BPS group and LP group, scale bar: $100 \mu \mathrm{m}$. A) In the health control group, the alveolar wall was thin, the alveolar cavity (arrow) was well inflated and there was no exudation in the alveolar cavity. B) In the PBS group, there was inflammatory exudation in alveolar cavity (arrow), and some air-filled alveoli were visible under the field of vision. C) In the LP group showing thickening lung septum, edema of alveolar wall (arrow), large amount of inflammatory exudate in lumen, and almost no inflatable alveoli under the field of vision. 
In this study, we also observed that swertianolin had no significant effect on the activation and proliferation of $\mathrm{T}$ cells, which may decrease the immunosuppressive function of MDSCs while improving the activity and proliferation of $\mathrm{T}$ cells.In recent years, people have made great efforts in order to research and discover bioactive natural medicines. This article clarifies the expression trajectory of MDSCs subgroups in sepsis and observes the effect of a natural product, swertianolin, on MDSCs at a cellular level. The limitation of this study is that the immunomodulatory function of the swertianolin is only studied in vitro, and more accurate effects need to be tested in vivo, which will be completed in our later study. Our team aims to continue conducting an in-depth research to clarify the target and mechanism of action of swertianolin in additional in vitro and in vivo assays.

\section{References}

1. Shankar-Hari M, Phillips GS, Levy ML, Seymour CW, Liu VX, Deutschman CS, et al. Developing a new definition and assessing new clinical criteria for septic shock: For the Third International Consensus Definitions for Sepsis and Septic Shock (Sepsis-3). JAMA 2016;315:775-87.

2. Lewis AJ, Rosengart MR. Bench-to-bedside: A translational perspective on murine models of sepsis. Surg Infect (Larchmt) 2018;19:137-41.

3. Boomer JS, To K, Chang KC, Takasu O, Osborne DF, Walton $\mathrm{AH}$, et al. Immunosuppression in patients who die of sepsis and multiple organ failure. JAMA 2011;306:2594-605.

4. Rosenthal MD. Persistent inflammatory, immunosup pressed, catabolic syndrome (PICS): A new phenotype of multiple organ failure. J Adv Nutr Hum Metab 2015;1:e784.

5. McPeak MB, Youssef D, Williams DA, Pritchett CL, Yao ZQ, McCall CE, et al. Frontline Science: Myeloid cell-specific deletion of Cebpb decreases sepsis-induced immunosuppression in mice. J Leukoc Biol 2017;102:191-200.

6. Hotchkiss RSD, Monneret GP, Payen DM. Immunosuppression in sepsis: a novel understanding of the disorder and a new therapeutic approach. Lancet Infect Dis 2013;13:260-8.

7. Bosurgi R. Sepsis: a need for new solutions. Lancet Infect Dis 2015;15:498-99.

8. Arens C, Bajwa SA, Koch C, Siegler BH, Schneck E, Hecker A, et al. Sepsis-induced long-term immune paralysis-results of a descriptive, explorative study. Crit Care 2016;20:93-9.

9. Fleming V, Hu X, Weber R, Nagibin V, Groth C, Altevogt P, et al. Targeting myeloid-derived suppressor cells to bypass tumor-induced immunosuppression. Front Immunol 2018;9:398.

10. Brudecki L, Ferguson DA, Yin D, Lesage GD, McCall CE, El Gazzar M. Hematopoietic Stem-progenitor cells restore immunoreactivity and improve survival in late sepsis. Infect Immun 2012;80:602-11.

11. Nagaraj S, Gabrilovich DI. Myeloid-derived suppressor cells as regulators of the immune system. Nar Rev Immunol 2009;9:162-74.

12. Zeng Q, Yang B, Sun H, Feng G, Jin L, Zou Z, et al. Myeloidderived suppressor cells are associated with viral persistence and downregulation of TCR $\zeta$ chain expression on CD8+ T cells in chronic hepatitis C patients. Mol Cells 2014;37:66-73.

13. Movahedi K, Guilliams M, Van den Bossche J, Van den Bergh $\mathrm{R}$, Gysemans C, Beschin A, et al. Identification of discrete tumor-induced myeloid-derived suppressor cell subpopulations with distinct $\mathrm{T}$ cell-suppressive activity. Blood 2008;111:4233-44.
14. Uhel F, Azzaoui I, Gregoire M, Pangault C, Dulong J, Tadie JM, et al. Early expansion of circulating granulocytic myeloidderived suppressor cells predicts development of nosocomial infections in patients with sepsis. Am J Respir Crit Care Med 2017; 196:315-27.

15. Nagaraj S, Youn J, Weber H, Iclozan C, Lu L, Cotter MJ, et al. Anti-inflammatory triterpenoid blocks immune suppressive function of MDSCs and improves immune response in cancer. Clin Cancer Res 2010;16:1812-23.

16. Qu P, Wang L, Lin PC. Expansion and functions of myeloidderived suppressor cells in the tumor microenvironment. Cancer Lett 2015;380:253-6.

17. Veglia F, Perego M, Gabrilovich D. Myeloid-derived suppressor cells coming of age. Nat Immunol 2018;19:108-19.

18. Wu X, Gu Y, Li L. The anti-hyperplasia, anti-oxidative and anti-inflammatory properties of Qing Ye Dan and swertiamarin in testosterone-induced benign prostatic hyperplasia in rats. Toxicol Lett 2017;265:9-16.

19. Zhang L, Cheng Y, Du X, Chen S, Feng X, Gao Y, et al. Swertianlarin, an herbal agent derived from Swertia mussotii Franch, attenuates liver injury, inflammation, and cholestasis in common bile duct-ligated rats. Evid Based Complement Alternat Med 2015;2015:948376.

20. Tang H, Ke Y, Ren Z, Lei X, Xiao S, Bao T, et al. Bioinformatics analysis of differentially expressed genes in hepatocellular carcinoma cells exposed to Swertiamarin. J Cancer 2019;10:6526-34.

21. He J, Tian C, Ouyang H, Adelakun TA, Yu B, Chang Y, et al. Determination of swertianolin in rat plasma by LC-MS/MS and its application to a pharmacokinetic study. Biomed Chromatogr 2014;28:1418-22.

22. Tian C, Zhang T, Wang L, Shan Q, Jiang L. The hepatoprotective effect and chemical constituents of total iridoids and xanthones extracted from Swertia mussotii Franch. J Ethnopharmacol 2014;154:259-66.

23. Wani BA, Ramamoorthy D, Rather MA, Arumugam N, Qazi AK, Majeed R, et al. Induction of apoptosis in human pancreatic MiaPaCa-2 cells through the loss of mitochondrial membrane potential (DeltaPsim) by Gentiana kurroo root extract and LC-ESI-MS analysis of its principal constituents. Phytomedicine 2013;20:723-33.

24. Gabrilovich DI, Nagaraj S. Myeloid-derived suppressor cells as regulators of the immune system. Nat Rev Immunol 2009;9:162-74.

25. Bronte V. Myeloid-derived suppressor cells in inflammation: Uncovering cell subsets with enhanced immunosuppressive functions. Eur J Immunol 2009;39:2670-2.

26. Condamine T, Gabrilovich DI. Molecular mechanisms regulating myeloid-derived suppressor cell differentiation and function. Trends Immunol 2010;32:19-25.

27. Cao T, Geng C, Ma Y, He K, Zhou N, Zhou J, et al. Chemical constituents of Swertia delavayi and their anti-hepatitis B virus activity. Zhongguo Zhong Yao Za Zhi 2015;40:897-902.

28. Hotchkiss RS, Moldawer LL, Phimister EG. Parallels between cancer and infectious disease. N Engl J Med 2014;371:380-3.

29. Ward PA, Rittirsch D, Flierl MA, Huber-Lang MS. Immunodesign of experimental sepsis by cecal ligation and puncture. Nat Protoc 2008;4:31-6.

30. Nascimento DC, Melo PH, Piñeros AR, Ferreira RG, Colón DF, Donate PB, et al. IL-33 contributes to sepsis-induced longterm immunosuppression by expanding the regulatory $\mathrm{T}$ cell population. Nat Commun 2017;8:389-95.

31. Janols H, Bergenfelz C, Allaoui R, Larsson A, Rydén L, Björnsson S, et al. A high frequency of MDSCs in sepsis patients, with the granulocytic subtype dominating in gram- 
positive cases. J Leukoc Biol 2014;96:685-93.

32. Richard S. Hotchkiss MD. The pathophysiology and treatment of sepsis. N Engl J Med 2003;11:273-8.

33. Riviello ED, Sugira V, Twagirumugabe T. Sepsis research and the poorest of the poor. Lanvet Infect Dis 2015;15:501-3.

34. Rosenthal MD, Moore FA. Persistent inflammation, immunosuppression, and catabolism: Evolution of multiple organ dysfunction. Surg Infect 2016;17:167-72.

35. Benjamim CF. Reversal of long-term sepsis-induced immunosuppression by dendritic cells. Blood 2005;1:127-31.

36. Deutschman CS, Tracey KJ. Sepsis: Current dogma and new perspectives. Immunity 2014;40:463-75.

37. Delano MJ, Ward PA. Sepsis-induced immune dysfunction: can immune therapies reduce mortality? J Clin Invest 2016;126:23-31.

38. Shao R, Fang Y, Yu H, Zhao L, Jiang Z, Li CS. Monocyte programmed death ligand-1 expression after 3-4 days of sepsis is associated with risk stratification and mortality in septic patients: a prospective cohort study. Crit Care 2016;20:124.

39. Goodwin AJ, Rice DA, Simpson KN, Ford DW. Frequency, cost, and risk factors of readmissions among severe sepsis survivors. Crit Care Med 2015;43:738-46.

40. Lv R, Zhao J, Lei M, Xiao D, Yu Y, Xie J. IL-33 Attenuates sepsis by inhibiting IL-17 receptor signaling through upregulation of SOCS3. Cell Physiol Biochem 2017;42:1961-72.

41. Mira JC, Gentile LF, Mathias BJ, Efron PA, Brakenridge SC, Mohr AM, et al. Sepsis pathophysiology, chronic critical illness, and persistent inflammation-immunosuppression and catabolism syndrome. Crit Care Med 2017;45:253-62.

42. Brudecki L, Ferguson DA, Yin D, Lesage GD, McCall CE, El Gazzar M. Hematopoietic stem-progenitor cells restore immunoreactivity and improve survival in late sepsis. Infect Immun 2012;80:602-11.

Received for publication: 21 June 2021. Accepted for publication: 26 July 2021.

This work is licensed under a Creative Commons Attribution-NonCommercial 4.0 International License (CC BY-NC 4.0).

(C) Copyright: the Author(s), 2021

Licensee PAGEPress, Italy

European Journal of Histochemistry 2021; 65:3292

doi:10.4081/ejh.2021.3292 